\title{
Rapid Eye Movements (REMs) and visual dream recall in both congenitally blind and sighted subjects
}

Helder Bértolo, Tiago Mestre, Ana Barrio, Beatriz Antona 


\title{
Rapid Eye Movements (REMs) and visual dream recall in both congenitally blind and sighted subjects
}

\author{
Helder Bértolo*a $^{* a}$ Tiago Mestre ${ }^{\mathrm{b}}$, Ana Barrio ${ }^{\mathrm{c}}$, Beatriz Antona ${ }^{\mathrm{c}}$ \\ aSEC Lisboa - Instituto Superior de Educação e Ciências, Campus Lumiar, Alameda das Linhas de \\ Torres, 179, 1750-142 Lisboa, Portugal; \\ ${ }^{\mathrm{b}}$ The Ottawa Hospital Research Institute / Ottawa Brain and Mind Research Institute, Cvic Campus, \\ 1053 Carling Avenue, Rm 2174a, Ottawa, ON, K1Y 4E9 Canada; \\ 'Facultad de Óptica y Optometría, Universidad Complutense de Madrid, Avda. Arcos de Jalón, 118, \\ 28037 Madrid, Spain
}

\begin{abstract}
Our objective was to evaluate rapid eye movements (REMs) associated with visual dream recall in sighted subjects and congenital blind.

During two consecutive nights polysomnographic recordings were performed at subjects home. REMs were detected by visual inspection on both EOG channels (EOG-H, EOG-V) and further classified as occurring isolated or in bursts. Dream recall was defined by the existence of a dream report. The two groups were compared using $t$-test and also the two-way ANOVA and a post-hoc Fisher test (for the features diagnosis (blind vs. sighted) and dream recall (yes or no) as a function of time).

The average of REM awakenings per subject and the recall ability were identical in both groups. CB had a lower REM density than CS; the same applied to REM bursts and isolated eye movements. In the two-way ANOVA, REM bursts and REM density were significantly different for positive dream recall, mainly for the CB group and for diagnosis; furthermore for both features significant results were obtained for the interaction of time, recall and diagnosis; the interaction of recall and time was however, stronger.

In line with previous findings the data show that blind have lower REMs density. However the ability of dream recall in congenitally blind and sighted controls is identical. In both groups visual dream recall is associated with an increase in REM bursts and density. REM bursts also show differences in the temporal profile. REM visual dream recall is associated with increased REMs activity.
\end{abstract}

Keywords: Visual imagery, Visual Perception, Blindness, Dreams, Dream Recall, REMs, EEG

\section{DREAM AND REM SLEEP.}

Dream emerges, since the very first primordial descriptions, with a close connection to REM sleep ${ }^{1}$.

The dissociation between the sleep and dreams is nowadays well known, since there are dream-like experiences in NREM sleep phases and REM sleep without dreams.

Different modelling mechanisms explain this dissociation between dream and REM sleep. Dream is controlled by dopaminergic mechanisms ${ }^{2}$ and REM sleep by cholinergic mechanisms of the brainstem ${ }^{3,4,5,6,7,8}$.

\subsection{Dream recall}

The study of dreams is closely related to the ability to recall them. There are different methods to study the capacity for dream recall: the morning recall by means of brief questionnaires or dream diaries or the recall after dream interruption. Both methods, if applied to frequent dreamers, revealed consistent results ${ }^{9,10}$. However, this consistency does not occur in infrequent dreamers, for whom the capacity for recall is greater in provoked awakenings ${ }^{10}$.

\footnotetext{
* helder.bertolo@iseclisboa.pt; www.iseclisboa.pt
}

Third International Conference on Applications of Optics and Photonics, edited by Manuel F. M. Costa, Proc. of SPIE Vol. 10453, 104532C · @ 2017 SPIE · CCC code: 0277-786X/17/\$18 · doi: 10.1117/12.2276048 
Dream recall capacity depends on the sleep phase, being higher in REM sleep than in $2 \mathrm{NREM}^{11}$. Indeed, recall frequency reaches $70-90 \%$ in REM awakenings, and only $5-10 \%$ in 2NREM awakenings 9 .

Recall frequency also varies with the recordings location, being lower in at-home recordings $(60 \%)^{13}$ than in recordings performed in a sleep lab $(91 \%)^{10}$.

Personality characteristics do not significantly influence dream recall ${ }^{9}$, although it does increase with interest, motivation, moodiness and stress ${ }^{14}$. The capacity for dream recall is also slightly higher in women.

The content of dream recall varies throughout the night. At the beginning of the night daily residues, related to every day's activity predominate, whereas later in the night hallucinatory or complex character narrative is more frequent ${ }^{11}$.

\subsection{Oneiric dream content}

Recall is also positively related to the vivacity, bizarreness and emotional content of dreams, which in turn are influenced by the oneiric visual content ${ }^{15}$.

In fact, the richness and continuity of the visual content increases the capacity for spontaneous recall ${ }^{16}$.

For these reasons the recall/visual content binomial assumes a particular relevance in the dreams of blind subjects. The works that consider that blind subjects do not have visual dream contents ${ }^{17}$, were contradicted by several studies, namely by one from our group ${ }^{18}$, which demonstrated that blind people do have the ability to draw their own dreams and, furthermore, showed negative correlations in the occipital area between visual content and EEG alpha power.

Additionally, favouring these results and suggesting the possibility of visual content, there are descriptions of rapid eye movements (REMs) in blind subjects, since in sighted subjects' dreams are associated with the presence of rapid eye movements ${ }^{19,20,21,22,23}$. However the controversy persists.

Thus, from a perspective of analysis of the relations between dream recall and brain or oculography activities, it is important to evaluate the changes associated with the presence of recall in blind and sighted subjects.

\section{REMS AND PGO WAVES}

Activation of the visual system during REM sleep is observed in EEG recordings, obtained with depth electrodes in animals, through the existence of spiky waves called PGO waves (Ponto-Geniculate-Occipital) because they are recorded in the reticular formation of the Pons, near the abducens nucleus, in the dorsal lateral geniculate nucleus and in the occipital cortex. PGO waves originate in the pons and project to the lateral geniculate nucleus and to the occipital cortex by separate anatomic pathways ${ }^{24,25}$.

PGO waves announce the onset of REM sleep, occurring within the minute before the onset of REM sleep electrographically defined. They provide an excellent example of non-random excitation of the forebrain during REM sleep originating from the brainstem. PGO waves carry information about the direction of rapid eye movements that will begin a few milliseconds after the onset of the PGO wave. The PGO wave in the left lateral geniculate nucleus is larger when the ocular movement is to the left and vice versa. The same directional specificity is found in PGO waves in the visual cortex.

Cell recordings provided important information about PGO waves generation, transmission, and effects in target areas. Intracellular recordings in the medial pontine reticular formation and in the adjacent tegmental reticular nucleus indicate the presence of neurons with long-lead discharges prior to PGO waves. These neurons satisfy correlation criteria for the generation of PGO waves $^{26}$.

Extracellular records in the area of the superior cerebellar peduncle (pedunculopontine nucleus and pons-midbrain junction) show PGO neurons that have a short-lead discharge pattern previous to the ipsilateral PGO waves of the lateral geniculate nucleus. These synchronous discharges are almost always perfectly correlated with those PGO waves, and 
both anatomical and antidromic reaction data and anatomical studies indicate projections to the lateral geniculate nucleus. For this reason, it is likely that these neurons are output neurons for brainstem-to-forebrain transmission of PGO waves.

Recordings from the main cells of the lateral geniculate nucleus and neurons in the occipital cortex indicate that PGO waves are associated with neuronal excitation $25,26,27,28$.

Furthermore, autoradiographic studies using 2-deoxyglucose techniques have demonstrated significant increases in glucose metabolism during REM sleep in the visual cortex, presumably as a consequence of intense neuronal activation $^{29,30}$.

This input into the visual system can be a substrate for the construction of visual experiences during the dream.

Since PGO waves predict the direction of the next eye movement, it has been hypothesized that they may represent an activation by REM sleep of the corollary discharge system for ocular movements while the rest of the brain is alerted to the following eye movement ${ }^{27,28,31}$.

During wakefulness, the corollary discharge system tells the rest of the brain to react to the changes in the retina, corresponding to the ocular movement, with a compensation in the subjective visual world, which is of the same magnitude but with opposite direction. As the "input" from the outer world is blocked during REM sleep, this compensation of the corollary discharge is itself perceived as a movement in the visual world. The authors propose this may be the source of some of the alterations in REM visual images.

The proposed sequence would be as follows:

REM System Activation (saccadic movements, horizontal first) $\rightarrow$ Corollary discharge system activation $\rightarrow$ Subjective perception of visual movement $\rightarrow$ Movement integration within the dream

This activation of the corollary discharge system without actual eye movements and without alteration of the visual world, leading to a subjective (or illusory) sensation of movement, has been demonstrated in experiments with wake human beings whom were induced a transient paralysis of the eye movements. A related alternative conceptualization is that the excitation of the forebrain caused by the PGO waves can interrupt the cognitive processes during dream, producing discontinuities or bifurcations and consequent oneiric weirdness ${ }^{32}$.

\subsection{PGOs in humans}

During a clinical neurosurgical operation to treat Parkinson's Disease symptoms, an invasive study into human PGO waves was performed ${ }^{33}$. Results indicated that PGO waves are a feature of human REM sleep, that they are generated or propagated in the pontomesencephalic tegmentum, that they are only partially associated with eye movements, and that they are associated with characteristic changes in cortical activity. Nonetheless, the concluding remarks of the study were that the Activation Synthesis Hypothesis ${ }^{7}$ that supports this interpretation of PGO wave function was largely supported.

Another study was conducted using a similar cohort of patients with Parkinson's Disease, with an expanded sample of 12 subjects $^{34}$. The conclusions were that subthalamic PGO-like waves can be recorded during pre-REM and REM sleep in humans and that the subthalamic nucleus may play an active role in an ascending activating network implicated in the transmission of PGO waves during REM sleep in humans. Much like feline models, PGO wave clusters were observed to be closely related to REM Sleep and additionally demonstrated an observable fast oscillatory subthalamic beta activity in the 13-35 Hz range. Importantly, this study also demonstrated a degree of homogeneity between feline and human PGO wave function, in finding that PGO wave singlets precede REM Sleep onset by 30-90 s in both species.

A recent single-neuron study was conducted, using depth-electrode EEG, in a cohort of 13 epilepsy patients ${ }^{35}$. The authors were attempting to answer the question: Are REMs in sleep associated with visual-like activity, as during wakefulness? During sleep and wakefulness, REM onsets are associated with distinct intracranial potentials, reminiscent of ponto-geniculate-occipital waves. Individual neurons, especially in the MTL, exhibit reduced firing rates before 
REMs as well as transient increases in firing rate immediately after, similar to activity patterns observed upon image presentation during fixation without eye movements. Moreover, the selectivity of individual units is correlated with their response latency, such that units activated after a small number of images or REMs exhibit delayed increases in firing rates. Finally, the phase of theta oscillations is similarly reset following REMs in sleep and wakefulness, and after controlled visual stimulation. The results indeed suggest that REMs during sleep rearrange discrete epochs of visual-like processing as during wakefulness.

There have been very few attempts to find non-invasive evidence for PGO waves in humans. Nonetheless, the few that were performed have shown promise.

The only non-invasive imaging technology that has both the temporal and spatial resolution to potentially isolate and measure deep brain activity with sufficient fidelity to produce the characteristic electrophysiological PGO wave shape, is at present the magnetoencephalography $(\mathrm{MEG})^{36}$.

However, many other non-invasive technologies, such as fMRI and positron emission tomography (PET) are capable of playing important roles in providing indirect evidence to support the existence of this phenomenon, for instance through recording of general brain activity time-locked to REM. Such observations may contribute to understanding the nature and extent of the PGO wave generating network, their respective overlap with REM generation sites and the relationship with limbic regions such as the Amygdala ${ }^{37}$.

A study using positron emission tomography and iterative cerebral blood flow measurements in humans confirmed that brain regions where PGO waves are most easily recorded in animals would be differentially more active in REM sleep than in wakefulness, in relation with the density of REMs production ${ }^{38}$. Accordingly, they found a significant interaction effect in the right geniculate body and in the primary occipital cortex. The result supports the hypothesis of the existence of processes similar to PGO waves in humans, responsible for REM generation.

Like the work by Miyauchi and colleagues ${ }^{37}$, the study conducted by Peigneux and colleagues ${ }^{38}$ showed no significant activity during waking self-directed saccades, supporting the hypothesis that PGO waves play an important role in the generation of visual content that REM saccades subsequently process.

\subsection{Visual activation (and REMs) in blind subjects}

Visual activation during sleep in blind subjects is still subject to a strong debate. It is expected that blind subjects who have not lost their visual memories maintain the capacity for visual cortex activation.

The accounts of subjects who lost their vision later in life are very similar to those of the sighted, with reports of shapes, colours, movement... Moreover, when asked to imagine any object, their eyes have movements, as if to imagine the object it was necessary to scan $\mathrm{it}^{43}$.

The big question is to know what happens when you start loosing those memories, particularly when those memories do not exist at all, as is the case with the congenitally blind.

What are the dreams of an individual who lives permanently without light or inside a cave like? Will they be coloured, will they have shapes? These are the issues that arise in relation to the blind from birth. When a blind man is asked if he dreams the answer is immediate: "Yes!" But if we ask him if he sees anything in the dream, the answer is always doubtful because he does not know what it is to see ${ }^{44}$. Even if there were images and colours in his brain during the dream how could he recognize them? There is, therefore, no direct way, through the dream reports, to evaluate the presence of visual activation in the dream of congenitally blind subjects.

The study of eye movements during the dream of congenitally blind seemed at first to be the key to the resolution of the puzzle about the relationship between eye movements and the oneiric drama scenario. Is there a relationship? Initially, the hypothesis seemed to be confirmed since Berger failed to record ocular movements (with electro-oculography), in the dreams of congenitally blind people deprived of visual reports ${ }^{45}$. In fact, electro-oculography records the corneal-retinal potential, which may be absent if the retina is destroyed. Other methods, using mechanical sensors, have shown that the dreams of congenital blinds without visual reports have, after all, eye movements ${ }^{44}$. 
Our previous data ${ }^{18}$ do support Jouvet's hypothesis about dreaming in utero. He defended dream was also a tool to preserve the species' identity and that what we call "instinct" (e.g. the chick that breaks the egg and immediately starts eating the maize grains, without mother chicken having told it anything) is actually the result of dreaming with "basic" behaviours while in utero or in ovo.

While substantial work exists on postnatal visual development in children, little is known about the events during the prenatal stimulus scarce period, when intrinsic activity is expected to dominate. Responses to visual stimuli were reported from as early as the $28^{\text {th }}$ gestational week using neural electromagnetic activity measures acquired with $\mathrm{MEG}^{46}$. Nevertheless, foetal eye movements had been observed as early as $1981^{47}$.

Even-though the questions where functional visual activity occurs in utero, and how primary visual areas do respond to extrinsic stimuli are not yet answered, voxel based morphometry studies in congenitally blind subjects hint at brain areas that change due to the processing of extrinsic stimuli. While congenitally blind subjects exhibit lower cortical volume in both frontal areas (BA 44, 45) and visual areas (BA 17, 18) ${ }^{48}$, compared to a control population, functional connectivity between frontal areas (parts of BA 44, 45, and 47) and occipital areas is stronger in early blind ${ }^{49}$.

\section{REMS IN BLIND SUBJECTS}

\subsection{Methods}

We tested 20 voluntary adult subjects, 10 congenitally blind and 10 sighted, matched for sex, age and education, recording two consecutive nights of home PSG (polysomnographic) recordings, with serial awakenings for dream recall

Only stable REM stage awakenings were selected. All episodes occurring in NREM stage or including a transition from REM to another sleep stage were excluded. Hence, we got data from 10 congenitally blind and 8 sighted subjects.

Content analysis was performed on the dream reports using the Hall $\&$ Van de Castle definitions ${ }^{39}$ to code the different activities ${ }^{40}$.

Spectral analysis was performed with Fast Fourier Transforms on the 5-minute-long EEG recordings prior to each awakening for the different spectral rhythms (delta, theta, alpha, beta, sigma e gamma).

Visual inspection was also performed on those epochs to identify REMs on both EOG (electro-oculogram) channels: horizontal and vertical.

A REM was defined as a biphasic deflection with an amplitude higher than $30 \mathrm{mV}$ or monophasic deflection higher than $50 \mathrm{mV}$, both with a duration longer than 1 second.

REMs burts were formed by no less than 7 consecutive REMs with a 10-second period. All REMs not included in bursts were classified as isolated.

Subsequently, Total, Bursts and Isolated REMs density indexes were calculated. 


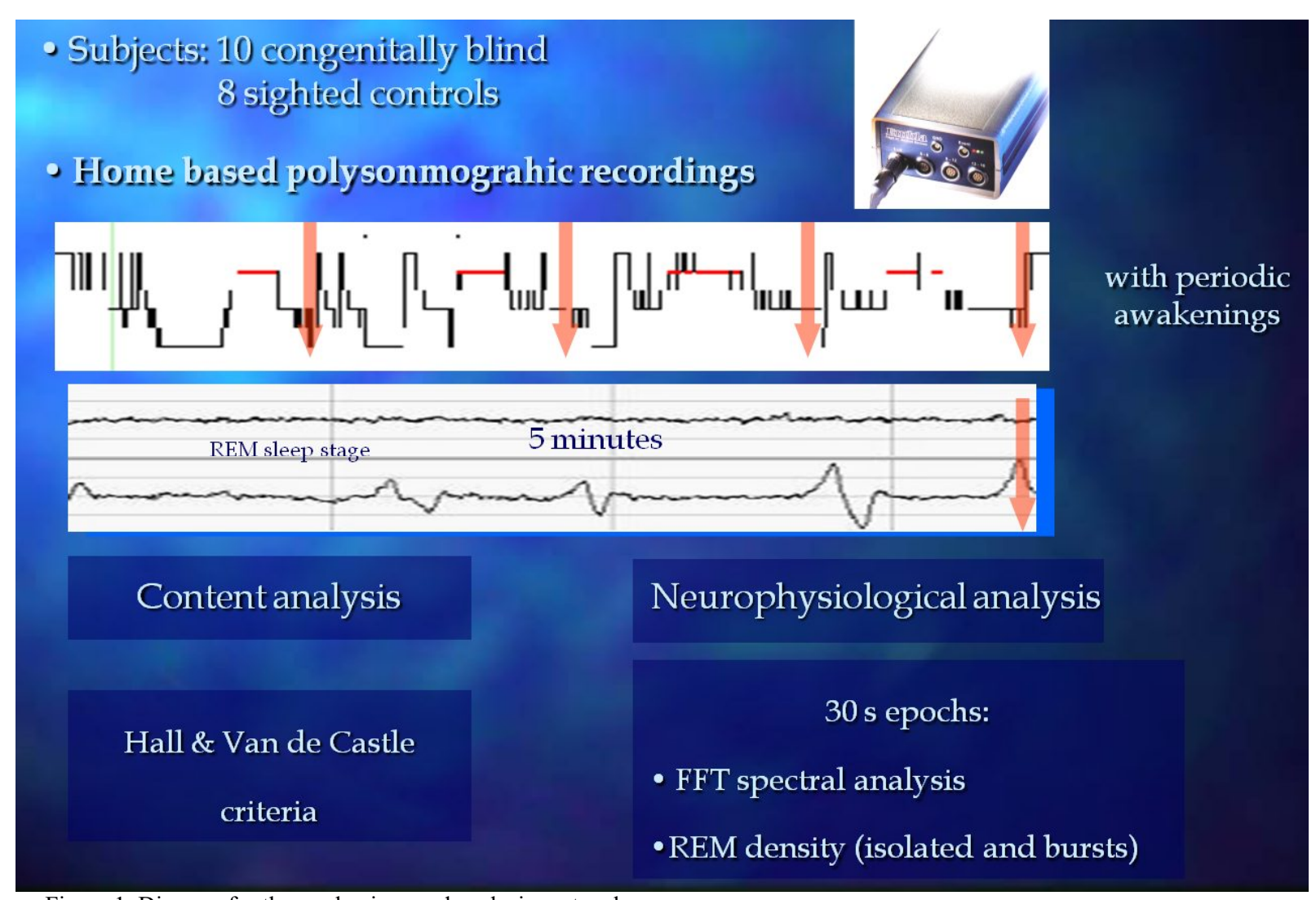

Figure 1. Diagram for the awakenings and analysis protocol.

The awakenings were divided according to the existence or non-existence of recall and the blind or sighted diagnosis. Based on these independent variables, statistical analyzes were performed, including variance analysis tests (ANOVA).

\subsection{Results}

Dream reports of the blind subjects were vivid with tactile, auditory and kinaesthetic references, but also with visual content. Neither the Global Activity Index (GAI) nor the Visual Activity Index (VAI) showed any difference between the two groups ${ }^{18}$.

The mean number of awakenings per subject and recall capacity were the same in both groups.

REM sleep awakenings were on average 1.6 for the congenitally blind (CB) and 1.7 for the control sighted (CS), both groups having a recall capacity of $60 \%$. Figure 2 shows the mean values and variances, and shows the comparison by percentiles between CB and CS.

There were differences regarding the density of MORs (total, bursts and isolated) between both groups (Figure 3).

There were no differences regarding the density of MORs and recall ability (Figure 4).

Blind subjects always present fewer REMs (Figure 5). 


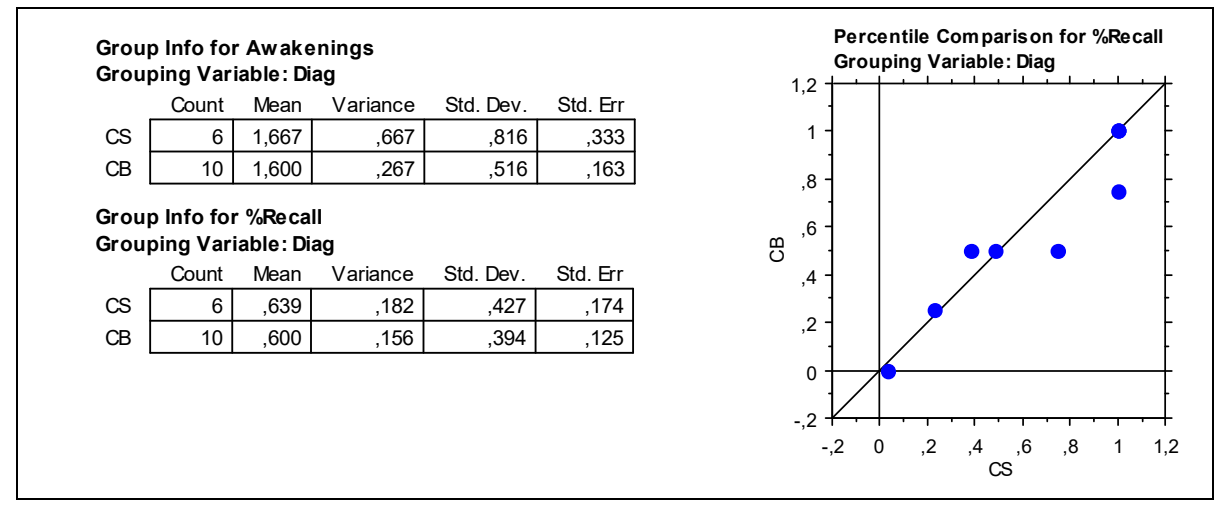

Figure 2. Awakenings and recall in both groups.

\begin{tabular}{|c|c|c|c|c|c|c|c|c|}
\hline & \multicolumn{3}{|c|}{ MEANS } & \multirow{2}{*}{$\begin{array}{l}\text { Mean2 } \\
\text { Effect }\end{array}$} & \multirow{2}{*}{$\begin{array}{l}\text { Mean2 } \\
\text { Error }\end{array}$} & \multirow{2}{*}{$\begin{array}{r}F(d f 1,2) \\
1,248\end{array}$} & \multirow[b]{2}{*}{ p } & \multirow[b]{2}{*}{ Signif? } \\
\hline & & CS & $\mathrm{CB}$ & & & & & \\
\hline \multirow{3}{*}{ REMs } & TOTAL & 8,7 & 1,9 & 2812,61 & 36,53 & 76,99 & 0,0000 & Yes \\
\hline & ISOLATED & 2,2 & 1,3 & 47,88 & 4,55 & 10,51 & 0,0013 & Yes \\
\hline & BURSTS & 6,5 & 0,6 & 2126,53 & 36,79 & 57,80 & 0,0000 & Yes \\
\hline
\end{tabular}

Figure 3. Differences for REMs density in both groups.

\begin{tabular}{|c|c|c|c|c|c|c|c|c|}
\hline & \multicolumn{3}{|c|}{ RECALL } & \multirow{2}{*}{$\begin{array}{l}\text { Mean2 } \\
\text { Effect }\end{array}$} & \multirow{2}{*}{$\begin{array}{l}\text { Mean2 } \\
\text { Error }\end{array}$} & \multirow{2}{*}{$\begin{array}{r}F(d f 1,2) \\
1,248\end{array}$} & \multirow[b]{2}{*}{$p$} & \multirow[b]{2}{*}{ Signif? } \\
\hline & & No & Yes & & & & & \\
\hline \multirow{3}{*}{ REMs } & TOTAL & 4,2 & 4,8 & 20,65 & 47,79 & 0,43 & $51,2 \%$ & - \\
\hline & ISOLATED & 1,5 & 1,7 & 2,17 & 4,74 & 0,46 & $50,0 \%$ & - \\
\hline & BURSTS & 2,7 & 3,1 & 9,44 & 45,33 & 0,21 & $64,9 \%$ & - \\
\hline
\end{tabular}

Figure 4. Differences for REMs with dream recall.

\begin{tabular}{rlrrrrrrr} 
& & \multicolumn{2}{c}{ RECALL } & Mean2 & Mean2 & F(df1,2) & \\
& & CS & CB & Effect & Error & $\mathbf{1 , 1 4 8}$ & $\boldsymbol{p}$ & Signif? \\
\hline \multirow{2}{*}{ REMs } & TOTAL & 8,6 & 2,3 & 1449,07 & 39,19 & 36,97 & $0,0 \%$ & Yes \\
& ISOLATED & 2,2 & 1,4 & 19,95 & 4,87 & 4,10 & $4,5 \%$ & Yes \\
& BURSTS & 6,5 & 0,9 & 1128,96 & 39,78 & 28,38 & $0,0 \%$ & Yes
\end{tabular}

Figure 5. Differences for REMs with dream recall in both groups.

\subsection{Analysis and Discussion}

Dream recall ability is similar in congenitally blind and sighted subjects, with an average of $60 \%$ rate in REM awakenings, in a protocol with in-home recordings, with provoked awakenings and no interaction with the researcher. 
Our results confirm the oneiric capacity of the blind subjects, contradicting earlier works that refute it ${ }^{41,42}$.

The fact that blind subjects present REMs and that these are correlated with visual dream recall is another result supporting our argument that they do activate visual areas during dream, being able to generate their own visual imagery.

We now know for a fact that PGO waves are not only related to REM but that they do predict the following eye movement direction $^{50}$. Moreover, studies in human subjects suggest that REMs are visually targeted eye movements commanded by the forebrain - in response to visual dream images and that REMs are involved in both the scanning and generation of dream imagery ${ }^{51}$.

There are differences in the functional anatomy of visually guided eye movements during waking and REM sleep. During wakefulness, top-down predictions about the proprioceptive and exteroceptive consequences of eye movements are sent to pontine and visual centres, respectively. The former elicit eye movements through classical reflex arcs (to suppress proprioceptive prediction error), while the latter anticipate the changes in retinal input. In sleep, there is a selective loss of precision on visual prediction errors. All this means is that the brain thinks its predictions in the visual domain are perfect, because they do not need correcting. This allows for perception without sensation; that is, dreaming ${ }^{52}$.

It looks like we only need to discover an example where all this happens without previous experience, since one thing is being able to dream and present REM without actually receiving inputs from the retina, visual imagery without visual perception, and another is being able to dream and present REM without ever having received those inputs, visual imagery without visual experience.

Schöpf and colleagues ${ }^{53}$ manage to do precisely that linking in utero eye movement with corresponding functional networks using BOLD resting-state fMRI data acquired from seven singleton foetuses between gestational weeks 30-36 with normal brain development. During the scan time, foetal eye movements were detected and tracked in the functional MRI data. Results showed that already in utero spontaneous foetal eye movements are linked to simultaneous networks in visual - and frontal cerebral areas. Data suggest that the preparation of the human visuomotor system links visual and motor areas already prior to birth.

Additionally, parietal-occipital activity was observed, which is known to be correlated with self-generated eye movements and plays a crucial role in encoding extrapersonal visual space. Since the foetuses were not presented with any visual stimuli these data may hint at the generator of intrinsic eye movements in human foetuses.

Hence, since foetuses present REMs, which are related with the exploration of the visual world, either during wakefulness and dreaming, and have never experienced visual stimuli, one may question what are they dreaming about? And if foetuses can dream, presumably with visual imagery, without ever having visual experience why does the same cannot happen with blind subjects.

Therefore, we propose that our results support the hypothesis that congenitally blind REMs during dream are equivalent to saccadic movements and consequently that they are able to generate visual imagery without visual experience.

\section{REFERENCES}

[1] Aserinsky, E. and Kleitman, N., "Regularly occurring periods of eye motility during sleep," Science 118, 273-274 (1953).

[2] Solms, M., [The Neuropsychology of dreams: a clinico-anatomic study], Erlbaum: Mahwah (1997).

[3] Hobson, J.A., Stickgold, R. and Pace-Schott, E.F., “The neurophysiology of REM sleep dreaming,” NeuroReport 9, R1-R14 (1998).

[4] Jouvet, M., "Recherches sur les structures nerveuses et les mecanismes responsables des differentes phases du sommeil physiologique," Archives Italiennes de Biologie 100, 125-206 (1962).

[5] McCarley, R.W. and Hobson, J.A., "Neuronal excitability modulation over the sleep cycle: A structural and mathematical model," Science 189, 58-60 (1975).

[6] Hobson, J.A., [The dreaming brain], Basic Books (1988). 
[7] Hobson, J.A. and McCarley, R.W., "The brain as a dream state generator: An activation-synthesis hypothesis of the dream process," Am. J. Psychiatry 134, 1335-1348 (1977).

[8] Pivik, R.T., McCarley, R.W. and Hobson, J.A., "Eye-movement-associated discharge in brain stem neurons during desynchronized sleep," Brain research 121, 59-76 (1977).

[9] Domhoff, G.W., [Finding Meaning in Dreams: A Quantitative Approach], Plenum Press, New York (1996).

[10] Murri, L., Massetani, R., Siciliano, G., Giovanditti, L. and Arena, R., "Dream recall after sleep interruption in brain-injured patients sleep," Sleep 8(4), 356-362 (1985).

[11] Guimarães, E., [Análise Psicofisiológica da Actividade Onírica], Master Thesis in Biophisics, Faculdade de Ciências de Lisboa, Lisboa (1996).

[12] Dement, W.C. and Kleitman, N., "The relation of the eye movements during sleep to dream activity. An objective method for the study of dreaming," Journal of Experimental Psychology 53, 339-346 (1957).

[13] Mestre, T., Bértolo, H. and Paiva, T., "Dream recall, REMs and spectral EEG components in blind and sighted," J. Sleep Res. 11(Suppl.1), 153 (2002).

[14] Zimmerman, W.B., "Sleep mentation and auditory awakening thresholds," Psychophysiology 6, 540-49 (1970).

[15] Kerr, N.H, "Dreaming, Imagery and Perception," [Principles and Practice of Sleep Medicine (3rd Ed.), 6(39)], Kryger M.H., Roth T., and Dement W.C. (Eds.), W.B. Saunders Company, 482-490 (2000).

[16] Butler, S. and Watson, R., "Individual differences in memory for dreams: the role of cognitive skills," Percept. Mot. Skills 61, 823-828 (1985).

[17] Kerr, N.H., Foulkes, D. and Schmidt, M., "The structure of laboratory dream reports in blind and sighted subjects," Percep Phsycophysiology 23, 117-124 (1978).

[18] Bertolo, H., Paiva, T., Pessoa, L., Mestre, T., Marques, R., Santos, R., "Visual dream content, graphical representations and EEG alpha activiti in congenitally blind subjects," Cogn Brain Res 15, 277-284 (2003).

[19] Myauchi, S., Takino, R., Fukuda, H. and Torii, S., "Electrophsysiological evidence for dreaming: human cerebral potentials associated with rapid eye movement during REM sleep," Electroencephalographic Clinical Neurophysiology 66, 383-390 (1987).

[20] Niiyama, Y., Shimizu, T., Abe, M. and Hishikawa, Y., "Phasic EEG activities associated with rapid eye movements during REM sleep in man," Electroencephalographic Clinical Neurophysiology vol. 70(5), 396-403 (1988).

[21] Miyauchi, S., Takino, R. And Azakami, M., "Evoked Potentials during REM sleep reflect dreaming," Electroencephalografic Clinical Neurophysiology 76(1), 19-28 (1990).

[22] Berger, R.J. and Oswald, I., "Eye movements during active and passive dreams," Science 137, 601 (1962).

[23] Aserinsky, E. and Kleitman, N., "Two types of ocular motility ocurring in sleep," J. Appl. Physiology 8, 1-10 (1955).

[24] Steriade, M. And McCarley, R.W., [Brainstem Control of Wakefulness and Sleep], Plenum Press, New York (1990).

[25] Steriade, M., Gloor, P., Llinás, R.R., Lopes da Silva, F.H. and Mesulam, M.M., "Basic mechanisms of cerebral rhythmic activities,” Electroenceph. Clin. Neurophysiol. 76, 481-508 (1990).

[26] McCarley, R.W. and Ito, K., "Intracellular evidence linking medial pontine reticular formation neurons to PGO wave generation," Brain Res. 280, 343-348 (1983).

[27] McCarley, R.W. and Hobson, J.A., "Cortical unit activity in desynchronized sleep," Science 167, 901-903 (1970).

[28] McCarley, R.W., Benoit, O. and Barrionuevo, G., "Lateral geniculate nucleus unitary discharge in sleep and waking: State- and rate-specific aspects," J. Neurophysiol. 50, 798-818 (1983).

[29] Hubel, D.H. and Livingstone, M.S. "A comparison between sleeping and waking spontaneous and visually evoked activity in cat striate cortex examined by single cell recording and 2-deoxy-glucose autoradiography," Soc. Neurosci. Abstr. 6, 314 (1980).

[30] Livingstone, M.S. and Hubel, D.H., "Effect of sleep and arousal on the processing of visual information in the cat," Nature 291, 554-561 (1981).

[31] McCarley, R.W. and Hobson, J.A., "The form of dreams and the biology of sleep," [Hanbook of Dreams: Research, Theories and Application], Wolman, B.B. (Ed.), Van Nostrand Reinhold, New York, 76-130 (1979).

[32] Mamelak, A. and Hobson, J.A., "Dream bizarreness as the cognitive correlate of altered neuronal behavior in REM sleep,” J. Cog. Neurosci. 1, 201-222 (1989).

[33] Lim, A. S., Lozano, A. M., Moro, E., Hamani, C., Hutchison, W. D., Dostrovsky, J. O., Lang, A.E., Wennberg, R.A. and Murray B.J., "Characterization of REM-sleep associated ponto-geniculo-occipital waves in the human pons," Sleep 30, 823-827 (2007). 
[34] Fernández-Mendoza, J., Lozano, B., Seijo, F., Santamarta-Liébana, E., Ramos-Platón, M.J., Vela-Bueno, A., Fernández-González F., "Evidence of subthalamic PGO-like waves during REM sleep in humans: a deep brain polysomnographic study," Sleep 32, 1117-1126 (2009).

[35] Andrillon, T., Nir, Y., Cirelli, C., Tononi, G. and Fried, I., "Single-neuron activity and eye movements during human REM sleep and awake vision," Nature Communications 6, 7884 (2015).

[36] Gott, J.A., Liley, D.T. and Hobson, J.A., "Towards a Functional Understanding of PGO Waves," Frontiers in Human Neuroscience 11 (89), 1-12 (2017).

[37] Miyauchi, S., Misaki, M., Kan, S., Fukunaga, T., and Koike, T., "Human brain activity time-locked to rapid eye movements during REM sleep,” Exp. Brain Res. 192, 657-667 (2009).

[38] Peigneux, P., Laureys, S., Fuchs, S., Delbeuck, X., Degueldre, C., Aerts, J., , Delfiore, G., Luxen, A. and Maquet, P., "Generation of rapid eye movements during paradoxical sleep in humans," Neuroimage 14, 701-708 (2001).

[39] Hall C.S., and Van de Castle R., [The content analysis of dreams], Appleton-Century-Crofts, New-York (1966).

[40] Domhoff G.W., [Finding Meaning in Dreams: A Quantitative Approach], Plenum Press, New York, 213-273 (1996).

[41] Holzinger, B., "The dreams of the blind: in consideration of the congenital and adventitously blindness," J. Sleep Res. 9(Supplement 1), 83 (2000).

[42] Lavie, P., [The Enchanted World of Sleep], Yale University Press (1996).

[43] Jouvet, M., [Le Sommeil el le Rêve], Editions Odile Jacob, Paris (1992).

[44] Jouvet, M., [Le Château des Songes], Editions Odile Jacob, Paris (1992).

[45] Berger, R.J., Olley, P. and Oswald, I., "EEG and eye movements and dreams of the blind," Electroencephalogr.Clin.Neurophysiol. 13, 827-833 (1961).

[46] Eswaran, H., Lowery, C.L., Wilson, J.D., Murphy, P. and Preissl, H., "Functional development of the visual system in human fetus using magnetoencephalography,” Exp.Neurol. 190(Suppl.), S52-S58 (2004).

[47] Birnholz, J.C., "The development of human fetal eye movement patterns," Science 213, 679-681 (1981).

[48] Ptito, M., Schneider, F.C.G., Paulson, O.B. and Kupers, R., "Alterations of the visual pathways in congenital blindness," Exp.BrainRes. 187, 41-49 (2008).

[49] Liu, Y., Yu, C., Liang, M., Li, J., Tian, L., Zhou, Y., Qin, W., Li, K. and Jiang T., "Whole brain functional connectivity in the early blind," Brain 130, 2085-2096 (2007).

[50] Hobson, J.A. and Friston, K.J., "Waking and dreaming consciousness: Neurobiological and functional considerations," Progress in Neurobiology 98(1), 82-98 (2012).

[51] Hobson, J.A., Hong C.C.-H. and Friston, K.J., "Virtual reality and consciousness inference in dreaming," Frontiers in Psychology 1133(5), 1-18 (2014).

[52] Hobson, J.A. and Friston, K.J., "Waking and dreaming consciousness: Neurobiological and functional considerations," Progress in Neurobiology 98(1), 82-98 (2012).

[53] Schöpf, V., Schlegl, T., Jakab, A., Kasprian, G., Woitek, R., Prayer, D. and Langs, G., “The relationship between eye movement and vision develops before birth," Frontiers in Human Neuroscience 8 (775), 1-6 (2014). 